November 2, 2018

IFUP-TH 4/97

LBNL-39946

UCB-PTH-97/06

\title{
Consequences of a U(2) Flavour Symmetry
}

\author{
Riccardo Barbieri ${ }^{1}$, Lawrence J. Hall ${ }^{2}$ and Andrea Romanino ${ }^{1}$ \\ 1 Physics Department, University of Pisa \\ and INFN, Sez. di Pisa, I-56126 Pisa, Italy \\ ${ }^{2}$ Department of Physics and Lawrence Berkeley National Laboratory \\ University of California, Berkeley, California 94720, USA
}

\begin{abstract}
While solving the supersymmetric flavour problem, a $\mathrm{U}(2)$ flavour symmetry might be at the origin of the pattern of fermion masses and mixings. The consequences of this hypothesis are spelled out concerning the parameters of the CKM matrix and their observability in $B$ - and $K$-physics. The relevance of searching for lepton flavour violation and for the electric dipole moments of the electron and the neutron is also emphasized.
\end{abstract}

${ }^{*}$ This work was supported in part by the Director, Office of Energy Research, Office of High Energy and Nuclear Physics, Division of High Energy Physics of the U.S. Department of Energy under Contract DE-AC03-76SF00098, in part by the National Science Foundation under grant PHY-95-14797 and in part by the U.S. Department of Energy under contract DOE/ER/01545-700. This work was partially supported by the "Beyond the Standard Model" TMR Network under the EEC contract No. ERBFMRX-CT960090. 
1. Flavour physics is a promising area for discoveries and surprises. On the experimental side, a number of significant observables are likely to be measured in the coming years: CPasymmetries in $B$-decays are an example, which is obvious but, we hope, far from unique, as indicated below. On the theoretical front, a qualitative and quantitative explanation of the pattern of fermion masses and mixing angles is still elusive, despite considerable effort.

If nature is (approximately) supersymmetric, the flavour problem acquires a new aspect. In supersymmetric theories there are mass and interaction matrices for the squarks and sleptons, leading to a richer flavour structure. In particular, if fermions and scalars of a given charge have mass matrices which are not diagonalized by the same rotation, new mixing matrices, $W$, occur at gaugino and higgsino vertices. In turn, the Flavour Changing Neutral Current (FCNC) and/or CP-violating phenomena induced by these new interactions must not violate the corresponding experimental bounds.

In a given supersymmetric theory, these different aspects of the flavour problem may or may not be related to each other. A prevailing attitude, so far, has been to consider the flavour structure of the scalar mass matrices as being unrelated to the one of the fermion mass matrices: the flavour blind mechanism that generates the supersymmetry breaking scalar masses results in a sufficient degeneracy of the sfermions to keep the FCNC and CPviolating phenomena under control. Without discussing here in which cases this physical consequence is actually justified, in this work we take, as others have done [1], an opposite viewpoint: the flavour structure of the mass matrices of the fermions and of the scalars are related to each other by a symmetry principle. It is this principle which is at the origin of the pattern of fermion masses and mixings and is, at the same time, responsible for the sufficient suppression of FCNC and CP-violating phenomena.

As argued in previous works [2, 解, a $\mathrm{U}(2)$ flavour symmetry in which the lighter two generations transform as a doublet and the third generation as a trivial singlet, like the Higgs fields, emerges as a candidate to fulfill this role. There are simple reasons for that. In the limit of unbroken $\mathrm{U}(2)$, only the third generation of fermions can acquire a mass, whereas the first two generations of scalars are exactly degenerate. While the first property is not a bad approximation of the fermion spectrum, the second one is what one needs to keep under control FCNC and CP-violating phenomena. Furthermore, the rank 2 of U(2) allows a two step breaking pattern

$$
\mathrm{U}(2) \stackrel{\epsilon}{\rightarrow} \mathrm{U}(1) \stackrel{\epsilon^{\prime}}{\rightarrow} 0
$$

controlled by two small parameters $\epsilon$ and $\epsilon^{\prime}$, to be at the origin of the generation mass hierarchies $m_{3} \gg m_{2} \gg m_{1}$ in the fermion spectrum. Although it is natural to view 
$\mathrm{U}(2)$ as a subgroup of $\mathrm{U}(3)$, the maximal flavour group in the case of full intra-family gauge unification, $\mathrm{U}(3)$ will be anyhow strongly broken to $\mathrm{U}(2)$ by the large top Yukawa coupling.

2. To fully exploit the consequences of the $\mathrm{U}(2)$ flavour symmetry, its breaking pattern must be specified more precisely. As mentioned, the three generations of matter fields $\psi$ transform as $\mathbf{2} \oplus \mathbf{1}$,

$$
\psi=\psi_{a} \oplus \psi_{3} .
$$

Taking the Higgs bosons to be flavour singlets, the Yukawa interactions transform as: $\left(\psi_{3} \psi_{3}\right),\left(\psi_{3} \psi_{a}\right),\left(\psi_{a} \psi_{b}\right)$. Hence the only relevant $\mathrm{U}(2)$ representations for the fermion mass matrices are $1, \phi^{a}, S^{a b}$ and $A^{a b}$, where $S$ and $A$ are symmetric and antisymmetric tensors, and the upper indices denote a U(1) charge opposite to that of $\psi_{a} . \phi^{a}$, $S^{a b}$ and $A^{a b}$ can be viewed as "flavon" fields.

We make the simplifying assumption that each of these fields participate in only one stage of the symmetry breaking in (1). Since $A^{a b}$ alone would break U(2) down to $\mathrm{SU}(2)$, whereas it would break $\mathrm{U}(2)$ completely in association with $\phi^{a}$ and/or $S^{a b}$, it can only participate in the last stage of breaking in (11): $\mathrm{U}(1) \rightarrow 0$. Therefore, $A^{12}=-A^{21}=\mathcal{O}\left(\epsilon^{\prime}\right)$. On the other hand, to account for $\left|V_{c b}\right| \simeq m_{s} / m_{b}$ in term of a unique parameter $\epsilon$, both $\phi^{a}$ and $S^{a b}$ must participate in the first stage of breaking in (11): $\mathrm{U}(2) \rightarrow \mathrm{U}(1)$ ]. Hence, in the basis where $\phi^{2}=\mathcal{O}(\epsilon)$ and $\phi^{1}=0, S^{22}=\mathcal{O}(\epsilon)$ and all other components of $S$ vanish if they were non-zero they would break $\mathrm{U}(1)$ at order $\epsilon$, which is excluded by (11). We are thus led to Yukawa matrices in up, down and charged lepton sectors of the form:

$$
\left(\begin{array}{ccc}
0 & \epsilon^{\prime} & 0 \\
-\epsilon^{\prime} & \epsilon & \epsilon \\
0 & \epsilon & 1
\end{array}\right) .
$$

All non vanishing entries have unknown coefficients of order unity, while still keeping $\lambda_{12}=-\lambda_{21}$. With $\epsilon \simeq 0.02$ and $\epsilon^{\prime} \simeq 0.004$, such a pattern agrees qualitatively well with the observed quark and lepton masses and mixings, with a few exceptions which can be understood in terms of the composition of the Higgs which couple to the $D / E$ sectors and of the intra-generation structure of the Yukawa couplings [3].

The mass and interaction matrices for the scalars arising from supersymmetry breaking can be discussed along similar lines. We assume that the same representations, $\phi^{a}, S^{a b}$ and

${ }^{\dagger}$ Without introducing unnatural coefficients and/or cancellations, the two other alternatives, $S^{a b}=\mathcal{O}(\epsilon)$ and $\phi^{a}=\mathcal{O}\left(\epsilon^{\prime}\right)$, or viceversa, would lead to $\left|V_{c b}\right| \approx\left|V_{u s}\right| m_{s} / m_{b}$ or $\left|V_{c b}\right| \approx\left(m_{s} / m_{b}\right)^{1 / 2}$, respectively. 
$A^{a b}$, which play a role in the fermion Yukawa sector, are also relevant in the description of flavour breaking in the scalar sector. In this way, it is immediate to see that the " $A$ terms" have the same structure of the Yukawa matrices in (2), whereas the only terms of numerical relevance in the scalar mass matrices are those ones linear in $\phi^{a},\left(\phi^{a}\right)^{\dagger}, \phi^{a}\left(\phi^{b}\right)^{\dagger}$ and $S^{a b}\left(S^{b c}\right)^{\dagger}$. Hence, the resulting mass matrices have the form

$$
m^{2}=\left(\begin{array}{ccc}
m_{1}^{2} & 0 & 0 \\
0 & m_{1}^{2}\left(1+\epsilon^{2}\right) & \epsilon m_{4}^{2^{*}} \\
0 & \epsilon m_{4}^{2} & m_{3}^{2}
\end{array}\right)
$$

where $m_{1}, m_{3}$ and $m_{4}$ are masses of the order of the supersymmetry breaking scale.

3. The CKM matrix has many possible forms, as there are many ways to choose the three Euler angles. For example the original choice of Kobayashi and Maskawa took the form $V=$ $R_{23}\left(\theta_{23}\right) R_{12}\left(\theta_{12}\right) P(\delta) R_{23}^{T}\left(\theta_{23}^{\prime}\right)$, where $R_{i j}\left(\theta_{i j}\right)$ is a $2 \times 2$ rotation in the $i j$ plane by angle $\theta_{i j}$. $P$ is a diagonal phase matrix with non-zero entries $\left(1,1, e^{i \delta}\right)$. To appreciate the relationship between the CKM matrix and the quark masses there is a preferred choice for the Euler angles: the larger terms (perturbations) in the quark mass matrices should be diagonalized first. This suggests consideration of the form $V=R_{12}\left(\theta_{12}^{U}\right) R_{23}\left(\theta_{23}\right) P(\phi) R_{12}^{T}\left(\theta_{12}^{D}\right)$, where the diagonal phase matrix has entries $\left(e^{-i \phi}, 1,1\right)$. For the case that the 13,31 and 11 entries vanish, and the 12 and 21 entries have equal magnitudes, as in (2), a perturbative diagonalization requires first a 23 rotation, then a 12 rotation giving [4]

$$
V=\left(\begin{array}{ccc}
c_{12}^{D}+s_{12}^{D} s_{12}^{U} e^{i \phi} & s_{12}^{D}-s_{12}^{U} e^{i \phi} & -s_{12}^{U} s \\
s_{12}^{U}-s_{12}^{D} e^{i \phi} & c_{12}^{D} e^{i \phi}+s_{12}^{U} s_{12}^{D} & s \\
s_{12}^{D} s & -c_{12}^{D} s & e^{-i \phi}
\end{array}\right)
$$

where

$$
\begin{gathered}
s_{12}^{D}=\sqrt{\frac{m_{d}}{m_{s}}}\left(1-\frac{m_{d}}{2 m_{s}}\right), \\
c_{12}^{D}=\sqrt{1-\left(s_{12}^{D}\right)^{2}},
\end{gathered}
$$

and

$$
s_{12}^{U}=\sqrt{\frac{m_{u}}{m_{c}}},
$$

with $m_{u}$ and $m_{c}$, as $m_{d}$ and $m_{s}$, renormalized at the same scale. The biggest errors in (4) are in $V_{u b}$ and $V_{t d}$, of relative order $s\left(m_{d} m_{c} / m_{u} m_{s}\right)^{1 / 2}$ f and $s$ respectively.

\footnotetext{
$\ddagger$ The precise values of these correction depends on the Yukawa matrix elements $\lambda_{23}^{U, D}$ and $\lambda_{32}^{U, D}$. Taking $\left|\lambda_{23} / \lambda_{32}\right|^{U, D}=1$ at the unification scale and barring significant cancellations in the determination of $s$, the
} 
At first sight this is a cumbersome form for the CKM matrix, since $V_{u s}$ contains two terms with a relative phase: $V_{u s} \equiv s_{c} e^{-i \beta}=s_{12}^{D}-s_{12}^{U} e^{i \phi}$. However, this is nothing other than the well-known unitarity triangle, with $\phi=\alpha$ and $\beta$ the usual angles:

$$
\begin{gathered}
\alpha \equiv \arg \left(-\frac{V_{t b}^{*} V_{t d}}{V_{u b}^{*} V_{u d}}\right) \approx \phi \\
\beta \equiv \arg \left(-\frac{V_{c b}^{*} V_{c d}}{V_{t b}^{*} V_{t d}}\right) \approx \arg \left(1-\frac{s_{12}^{U}}{s_{12}^{D}} e^{-i \phi}\right) \\
\gamma \equiv \arg \left(-\frac{V_{u b}^{*} V_{u d}}{V_{c b}^{*} V_{c d}}\right) \approx \pi-\alpha-\beta .
\end{gathered}
$$

This can be seen by rephasing (4) into the form

$$
V \approx\left(\begin{array}{ccc}
1 & s_{c} & -s_{12}^{U} s \\
-s_{c} & 1 & s e^{-i(\alpha+\beta)} \\
s_{12}^{D} s & -s e^{i \beta} & e^{-i \alpha}
\end{array}\right)
$$

The orthogonality of the first and third columns, which gives the usual unitarity triangle, gives $s\left(s_{c} e^{-i \beta}-s_{12}^{D}+s_{12}^{U} e^{i \alpha}\right)=0$. In this form it is clear that $\beta$ is important for much kaon physics: for example, the $W$-box contribution to $\epsilon_{K}$ is proportional to $\sin (2 \beta)$.

The simple pattern of $\mathrm{U}(2)$ breaking implies that, given the masses of the four light quarks, all CKM matrix elements are known in terms of just two free parameters, $s=\left|V_{c b}\right|$ and $\phi$. Using the values for $\left|V_{u s}\right|,\left|V_{c b}\right|$ and $\left|V_{u b} / V_{c b}\right|$ listed in Table 1 , we can predict $\left|V_{t d} / V_{t s}\right|=\left(m_{d} / m_{s}\right)^{1 / 2}$ and the CP-violating phase $\phi$, as well as the angles $\beta$ and $\gamma$ of the unitarity triangle.

For $m_{c}$ and $m_{s}$, we use the values listed in Table 1, whereas the ratios of the light quark masses, $m_{u} / m_{d}$ and $m_{d} / m_{s}$ require a discussion. Second order chiral perturbation theory for the pseudoscalar meson masses determines, to a remarkable accuracy, the combination [5]

$$
Q=\frac{\frac{m_{s}}{m_{d}}}{\sqrt{1-\frac{m_{u}^{2}}{m_{d}^{2}}}}=22.7 \pm 0.08 .
$$

Additional assumptions, plausible but not following from pure QCD, lead to [5]

$$
m_{u} / m_{d}=0.553 \pm 0.043 .
$$

largest correction occurs in $\left|V_{u b} / V_{c b}\right|=\sqrt{m_{u} / m_{c}}$, and ranges from about 1 to $7 \%$, depending on relative phases. 


\begin{tabular}{||c|c||c|c||}
\hline$\left(m_{s}\right)_{1 \mathrm{GeV}}$ & $(175 \pm 55) \mathrm{MeV}$ & $\left|V_{u s}\right|$ & $0.221 \pm 0.002$ \\
$\left(m_{c}\right)_{m_{c}}$ & $(1.27 \pm 0.05) \mathrm{GeV}$ & $\left|V_{c b}\right|$ & $0.040 \pm 0.003$ \\
$\left(m_{b}\right)_{m_{b}}$ & $(4.25 \pm 0.15) \mathrm{GeV}$ & $\left|V_{u b} / V_{c b}\right|$ & $0.08 \pm 0.02$ \\
$\left(m_{t}\right)_{m_{t}}$ & $(165 \pm 10) \mathrm{GeV}$ & $\alpha_{s}\left(M_{Z}\right)$ & $0.117 \pm 0.006$ \\
\hline
\end{tabular}

Table 1: Values of the parameters used in the text.

Finally, a better determination of the scale of the light quark masses is possible if use is made of the $\mathrm{SU}(5)$ relations, valid at the unification scale,

$$
m_{b}=m_{\tau}, \quad m_{d} m_{s}=m_{e} m_{\mu}
$$

as illustrated in [3]. Given the different level of uncertainty and/or assumptions in these equations, we describe the results of 4 "combined fits" with different inputs for the light quark mass ratios: i) (10) only; ii) (10) and (11); iii) (10) and (12); iv) (10), (11) and (12).

Treating all errors as "gaussian", at 90\% C.L., we obtain the results shown in figs. 1 . In fig. 1a, the boundary obtained from the unitarity constraints on a general parametrization of the $V_{\text {CKM }}$ is also shown. No similar boundary is given in figs. $1 \mathrm{~b}, 1 \mathrm{c}$, since unitarity alone does not limit the CKM phase $\phi$. It is possible, on the contrary to constrain $\phi$, if one includes also the $\mathrm{CP}$ violating parameter in $K$ physics, $\epsilon_{K}$, and the $B_{d}$ mixing mass, as in most Standard Model fits. Using for the standard quantities which parametrize QCD uncertainties, $B_{K}=0.8 \pm 0.2$ and $\sqrt{B} f_{B}=(200 \pm 40) \mathrm{MeV}$, the result in fig. $1 \mathrm{~d}$ is obtained. As explained below, however, this last plot does not have general validity.

4. The diagonalization of the matrix (3) leads to highly degenerate scalars of the first two generations, $\left(m_{2}^{2}-m_{1}^{2}\right) / m^{2} \approx \epsilon^{2} \approx 10^{-3}$, with $m^{2}$ an average scalar mass squared. On the contrary, the scalars of the third generation are likely to have masses very different from their first and second generation partners, $\left(m_{3}^{2}-m_{1,2}^{2}\right) \approx m^{2}$.

By going to a diagonal basis both for (2) and (3), mixing matrices are generated in the gaugino interactions: $\left(\bar{f}_{L, R} W_{L, R} \tilde{f}_{L, R}\right) \tilde{g}$ for the fermions of given chirality, $\mathrm{L}$ or $\mathrm{R}$, and their superpartners. By appropriate phase redefinitions of the fermion and scalar fields, while keeping the form (2) of $V_{\mathrm{CKM}}$ and the mass eigenvalues real and positive, it is possible to write the six matrices $W_{L, R}^{U, D, E}$ for $U$-quarks, $D$-quarks and charged leptons in terms of two 


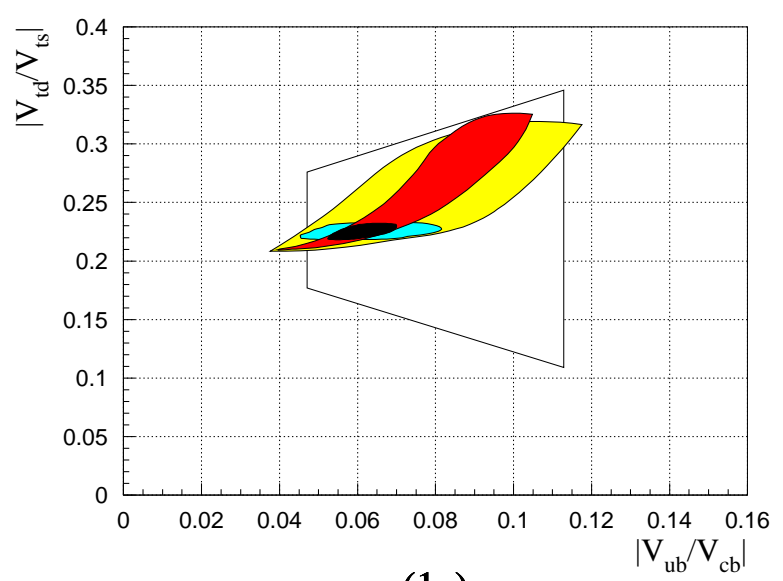

(1a)

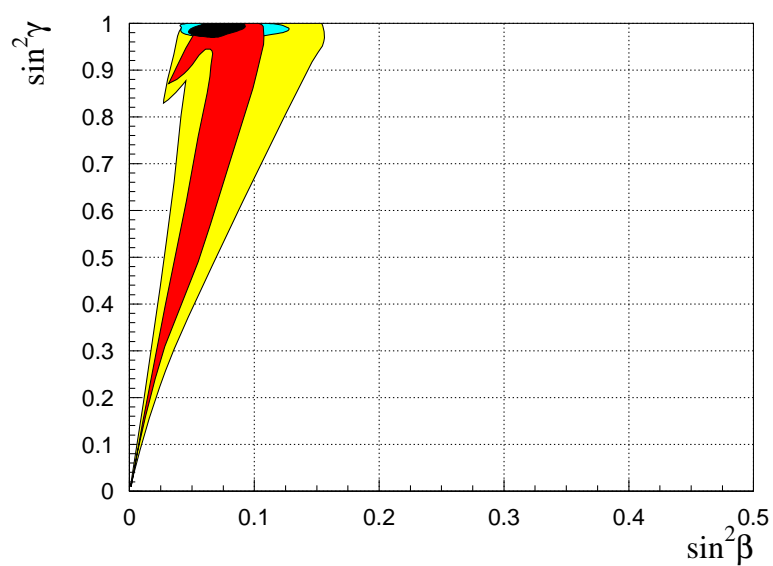

(1c)

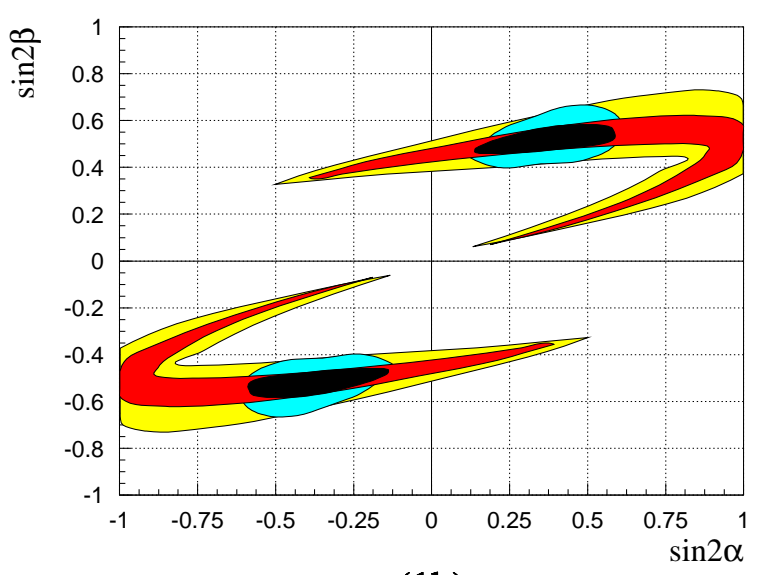

(1b)

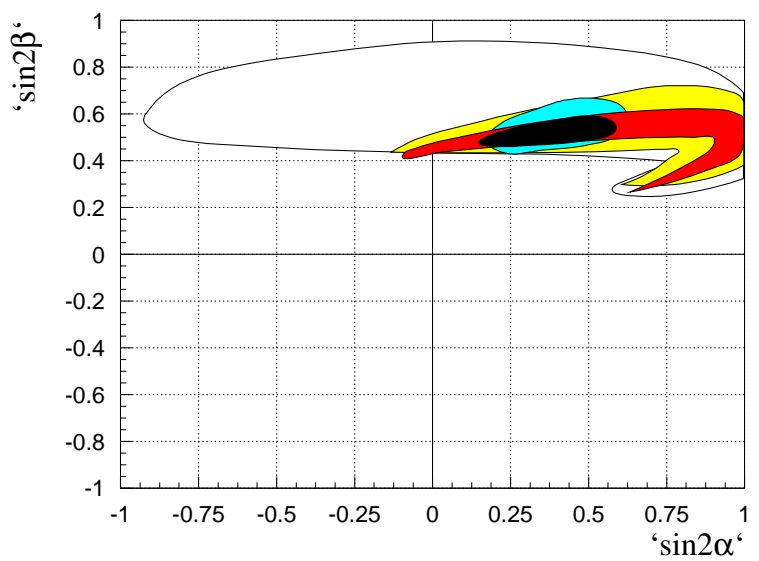

(1d)

Figure 1: $90 \%$ C.L. contours from the four combined fits defined in the text: i) larger, lighter area; ii) smaller, lighter area; iii) larger, darker area; iv) smaller, darker area. In figs. $1 \mathrm{a}$ and $1 \mathrm{~d}$ also shown is the contour for a general parametrization of the CKM matrix. Fig. $1 d$ is the only one to include $\epsilon_{K}$ and $\Delta m_{B_{D}}$ among the inputs. 
new parameters each, $s_{L, R}^{U, D, E}$ and $\gamma_{L, R}^{U, D, E}$

$$
\begin{gathered}
W_{L}^{U, D, E}=\left(\begin{array}{ccc}
c_{12} & -s_{12} & s_{12} s_{L} \\
s_{12} & c_{12} & -s_{L} \\
0 & s_{L} e^{i \gamma_{L}} & e^{i \gamma_{L}}
\end{array}\right)^{U, D, E} \\
W_{R}^{U, D, E}=\left(\begin{array}{ccc}
c_{12} & s_{12} & -s_{12} s_{R} \\
-s_{12} & c_{12} & -s_{R} \\
0 & s_{R} e^{i \gamma_{R}} & e^{i \gamma_{R}}
\end{array}\right)^{U, D, E}
\end{gathered}
$$

where $s_{12}^{U, D}$ have already been defined and, in analogy with them, $s_{12}^{E}=\sqrt{m_{e} / m_{\mu}}$. The parameters $s_{L, R}^{U, D, E}$ are all of order $\epsilon$. In this basis, the L-R mixings induced by the $A$-terms are still non diagonal and complex. They can, however, be treated as a perturbation except, maybe, for the $\tilde{t}_{L}-\tilde{t}_{R}$ mixing, which, in any event, does not alter the mixing matrices (13) and (14).

As already mentioned, via loops of supersymmetric particles, the $W$-matrices give rise to new FCNC and CP-violating phenomena. A close inspection shows that the most important effects occur in $\epsilon_{K}$, in $B-\bar{B}$ mixing, in the Electric Dipole Moments (EDM) of the electron and the neutron and, finally, in $\mu \rightarrow e \gamma$ and $\mu \rightarrow e$ conversion in atoms. In the case of $\epsilon_{K}$ and $B-\bar{B}$ mixing one obtains effects comparable to those present in the Standard Model. On the other hand, the effects in the dipole moments and in the Lepton Flavour Violating processes are at the level of the present experimental limits. The calculation of some typical, although partial, contributions to these observables gives in fact:

$$
\begin{gathered}
\epsilon_{K} \approx 2 \cdot 10^{-3}\left(\frac{500 \mathrm{GeV}}{m_{\tilde{q}}}\right)^{2}\left(\omega^{D}\right)^{2} \sin 2 \beta \\
\Delta m_{B_{d}} \approx 0.1 \mathrm{ps}^{-1}\left(\frac{500 \mathrm{GeV}}{m_{\tilde{q}}}\right)^{2} \omega^{D} \\
\operatorname{BR}(\mu \rightarrow e \gamma) \approx 2 \cdot 10^{-11}\left(\frac{100 \mathrm{GeV}}{m_{\tilde{l}}}\right)^{4}\left(\omega^{E}\right)^{2}\left(\frac{v_{2}}{v_{1}}\right)^{2} \\
d(e) \approx 6 \cdot 10^{-27} e \mathrm{~cm}\left(\frac{100 \mathrm{GeV}}{m_{\tilde{l}}}\right)^{2} \omega^{E} \sin \left(\gamma_{L}^{E}-\gamma_{R}^{E}\right)\left(\frac{v_{2}}{v_{1}}\right) \\
d(d) \approx 1 \cdot 10^{-26} e \mathrm{~cm}\left(\frac{500 \mathrm{GeV}}{m_{\tilde{q}}}\right)^{2} \omega^{D} \sin \left(\gamma_{L}^{D}-\gamma_{R}^{D}\right)\left(\frac{v_{2}}{v_{1}}\right)
\end{gathered}
$$

\footnotetext{
In equations (17) and (18) we consider the photino contribution. For the EDM of the $u$-quark one has $d(u) \approx 8 d(d)\left(v_{1} / v_{2}\right)^{2}\left(\omega^{U} / \omega^{D}\right)\left(\sin \left(\gamma_{L}^{U}-\gamma_{R}^{U}\right) / \sin \left(\gamma_{L}^{D}-\gamma_{R}^{D}\right)\right)$.
} 
where $d(e)$ and $d(d)$ denote the EDMs of the electron and the $d$-quark, respectively, $\omega^{U, D, E}=$ $\left(s_{L}^{U, D, E} s_{R}^{U, D, E}\right) / V_{c b}^{2}=\mathcal{O}(1)$ and $v_{2} / v_{1}$ is the ratio of the vacuum expectation values of the Higgs doublets.

In these equations, the effect of the splitting between the first two generations of scalars is neglected. This is completely justified for all the observables except $\epsilon_{K}$, in which case an additional effect of the same order of (15) is present, still proportional to $\sin 2 \beta$ as (15) and the SM contribution themselves. The effects are all due to the splitting $\left(m_{3}^{2}-m_{1,2}^{2}\right)$, taken large enough that the GIM suppression can be safely neglected. Therefore, $m_{\tilde{q}}$ in (15), (16) and (19) denotes the mass of the lightest $Q=1 / 3$ squark, whereas $m_{\tilde{l}}$ in (17) and (18) the mass of the lightest charged slepton, neglecting in both cases the difference between L- and R-states. The gluino mass in (15), (16), (19) and the photino mass in (17), (18) are taken equal to $m_{\tilde{q}}$ and $m_{\tilde{l}}$ respectively. Eq. (16) only includes the contribution of the $(V-A)(V+A)$ 4-quark operator. The $A$-terms are neglected in (17), (18), (19), whereas the " $\mu$-parameter" is taken equal to the relevant sfermion mass.

In spite of these limitations and uncertainties, on the basis of (17)-(19), we look with the greatest possible interest to any improvement of the experimental sensitivity in the search for $\mu \rightarrow e \gamma$ (or $\mu \rightarrow e$ conversion) $\Pi$ and for the electron and neutron EDMs.

5. In view of eq. (16), also the mixing and CP-violation in the $B$-system is likely to deviate from the expectation of the SM, as is the case for the parameter $\epsilon_{K}$. In particular, the experimental determination of the CKM parameters will be affected. For example, this explains why fig. 1d, which employs SM formulae for $\epsilon_{K}$ and $\Delta m_{B_{d}}$ as inputs, is not generally valid.

Precise formulae for the contribution from the gluino box diagram to the mixing matrix elements in $B_{d}-\bar{B}_{d}$ and $B_{s}-\bar{B}_{s}$ are

$$
\begin{gathered}
M_{12}\left(B_{d}\right)=\left(s_{12}^{D}\right)^{2}\left(-s_{L}^{D} s_{R}^{D} e^{i\left(\gamma_{L}^{D}+\gamma_{R}^{D}\right)} M_{L R}+\left(s_{L}^{D}\right)^{2} e^{2 i \gamma_{L}^{D}} M_{L L}+\left(s_{R}^{D}\right)^{2} e^{2 i \gamma_{R}^{D}} M_{R R}\right), \\
M_{12}\left(B_{s}\right)=s_{L}^{D} s_{R}^{D} e^{i\left(\gamma_{L}^{D}+\gamma_{R}^{D}\right)} M_{L R}+\left(s_{L}^{D}\right)^{2} e^{2 i \gamma_{L}^{D}} M_{L L}+\left(s_{R}^{D}\right)^{2} e^{2 i \gamma_{R}^{D}} M_{R R} .
\end{gathered}
$$

where $M_{L R}, M_{L L}, M_{R R}$, equal between $B_{d}$ and $B_{s}$ apart from $\mathrm{SU}(3)$-breaking effects, correspond to the $(V-A)(V+A),(V-A)(V-A),(V+A)(V+A)$ operators respectively. Using the vacuum insertion approximation and taking equal masses for right-handed and left-handed down squarks, $M_{L R}=4 M_{L L}=4 M_{R R}$.

\footnotetext{
I Lepton Flavour Violation could be inhibited by a suitable extension of the $\mathrm{U}(2)$ symmetry. This is not compatible, however, with intra-family gauge unification [6].
} 


\begin{tabular}{||c||c|c|c|c||}
\hline & i) & ii $)$ & iii $)$ & iv $)$ \\
\hline \hline $\mathrm{BR}\left(K^{+} \rightarrow \pi^{+} \nu \bar{\nu}\right) / 10^{-10}$ & $0.98_{-0.30}^{+0.40}$ & $0.83 \pm 0.20$ & $0.97_{-0.30}^{+0.50}$ & $0.84_{-0.22}^{+0.17}$ \\
\hline $\mathrm{BR}\left(K_{L} \rightarrow \pi^{0} \nu \bar{\nu}\right) / 10^{-10}$ & $0.22 \pm 0.13$ & $0.17 \pm 0.07$ & $0.20_{-0.10}^{+0.08}$ & $0.14 \pm 0.05$ \\
\hline
\end{tabular}

Table 2: Predictions for $K \rightarrow \pi \nu \bar{\nu}$ decays in the four "combined fits" described in the text.

Notice the relative minus sign in front of the presumably dominant LR-contributions to $B_{d}$ and $B_{s}$. This should lead to a significant deviation from the SM result (apart from $\mathrm{SU}(3)$-breaking effects)

$$
\frac{M_{12}\left(B_{d}\right)}{M_{12}\left(B_{s}\right)}=\left|\frac{V_{t d}}{V_{t s}}\right|^{2}
$$

as well as to the appearence of two different extra phases in the ratio of the mixing coefficients in $B_{d}$ and $B_{s}$

$$
\left(\frac{q}{p}\right)_{B_{d}} \equiv\left(\frac{V_{t b}^{*} V_{t d}}{V_{t b} V_{t d}^{*}}\right) e^{-2 i \phi_{B_{d}}}, \quad\left(\frac{q}{p}\right)_{B_{s}} \equiv\left(\frac{V_{t b}^{*} V_{t s}}{V_{t b} V_{t s}^{*}}\right) e^{-2 i \phi_{B_{s}}}
$$

How will it then be possible to test the predictions for the CKM parameters shown in figs. 1? Let us look at each of them, in turn.

Since we do not expect eq. (22) to be valid, the mixing in $B_{d}$ and $B_{s}$ might not be the right source of information on $\left|V_{t d} / V_{t s}\right|$. On the contrary, a clean way to measure $\left|V_{t d}\right|$ is provided by the branching ratio of $K^{+} \rightarrow \pi^{+} \nu \bar{\nu}$, which is not affected by gluino corrections. Our prediction is shown in Table 2 for the four combined fits. Also not affected by gluino corrections are the decay modes $B \rightarrow \chi_{s}\left(\chi_{d}\right) \nu \bar{\nu}$ and, to a good approximation, $B \rightarrow \chi_{s}\left(\chi_{d}\right) l^{+} l^{-}$. Therefore the ratios $\left(B \rightarrow \chi_{d}\right) /\left(B \rightarrow \chi_{s}\right)$ are another potential source of information on $\left|V_{t d} / V_{t s}\right|$, if $\mathrm{SU}(3)$-breaking effects, especially in $B \rightarrow \chi_{s}\left(\chi_{d}\right) l^{+} l^{-}$, can be kept under control.

The new phases $\phi_{B_{d}}$ and $\phi_{B_{s}}$ defined in (23) modify the CP asymmetries of neutral $B$-decays into $\mathrm{CP}$ eigenstates. For example, concentrating on the coefficient of the $\sin \Delta m t$ term in the time dependent CP asymmetries, one has [8]

$$
a_{C P}\left(B_{d} \rightarrow \psi K_{S}\right)=-\sin 2\left(\beta+\phi_{B_{d}}\right), \quad a_{C P}\left(B_{s} \rightarrow \psi \phi\right)=-\sin 2 \phi_{B_{s}}
$$

$\| B \rightarrow \chi_{s}\left(\chi_{d}\right)$ decays, as $\epsilon_{K}, B-\bar{B}$ mixing and $K \rightarrow \pi$ decays, receive also corrections due to charged Higgs and chargino-stop exchanges, controlled by the top Yukawa coupling, which are there in any supersymmetric extension of the Standard Model. These corrections are most prominent in $B \rightarrow$ $\chi_{s}\left(\chi_{d}\right) l^{+} l^{-}$, as in $B \rightarrow \chi_{s}\left(\chi_{d}\right) \gamma$, even for relatively small $v_{2} / v_{1}$ []]. Not seeing any effect in $B \rightarrow \chi_{s} l^{+} l^{-}$at $B$-factories would make this kind of correction negligible everywhere else. In any case, they do not affect $\frac{M_{12}\left(B_{d}\right)}{M_{12}\left(B_{s}\right)}$ nor the CP-asymmetries. 


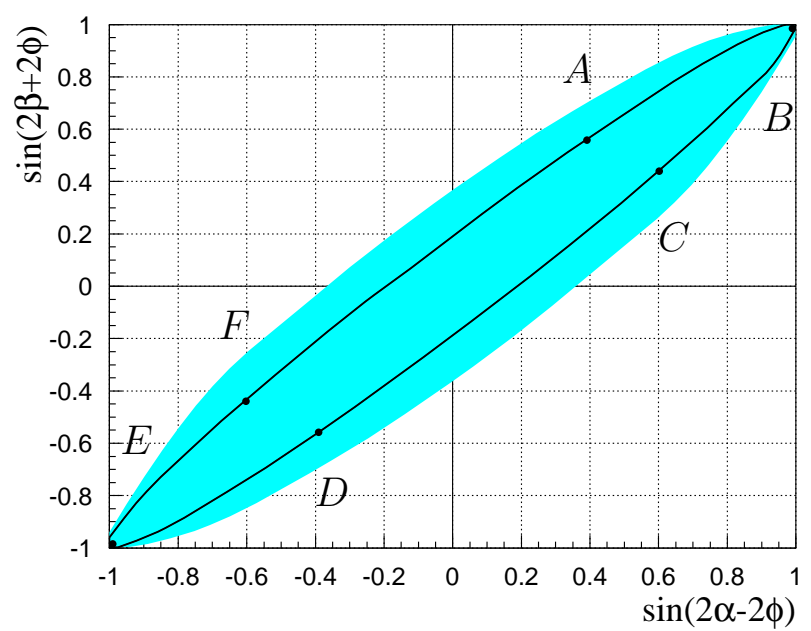

Figure 2: $90 \%$ C.L. contour for the asymmetries measurable in $B_{d} \rightarrow \psi K_{S}(-\sin 2(\beta+$ $\left.\left.\phi_{B_{d}}\right)\right)$ and $B_{d} \rightarrow \pi \pi\left(\sin 2\left(\alpha-\phi_{B_{d}}\right)\right)$ in fits ii) and iv $)$, which give indistinguishable results. $A, D: \phi=0, \pi / 2 ; B, E: \phi=\pi / 6,2 \pi / 3 ; C, F: \phi=\pi / 3,5 \pi / 6$.

or, neglecting possible penguin contributions or assuming their elimination by a proper isospin analysis,

$$
a_{C P}\left(B_{d} \rightarrow \pi \pi\right)=\sin 2\left(\alpha-\phi_{B_{d}}\right) .
$$

Therefore, $\sin 2 \alpha$ and $\sin 2 \beta$ are not directly accessible from the $\mathrm{CP}$ asymmetries in $B$ decays, nor is $\sin 2 \beta$ obtainable from $\epsilon_{K}$, although required to be non-vanishing due to (15). Yet a correlation between $a_{C P}\left(B_{d} \rightarrow \psi K_{S}\right)$ and $a_{C P}\left(B_{d} \rightarrow \pi \pi\right)$, as given in (24) and (25), can still be predicted at least if use is made of (11). This correlation is shown at $90 \%$ C.L. in fig. 2 for arbitrary values of $\phi_{B_{d}}$.

A clean way to get $\sin ^{2} \beta$ is again through $K$-decays. The branching ratio for $K_{L} \rightarrow \pi^{0} \nu \bar{\nu}$ is short distance dominated, it is not affected by gluino corrections and is proportional, through a rather precisely known coefficient [9], to $\left|V_{t d} V_{t s}\right|^{2} \sin ^{2} \beta . K_{L} \rightarrow \pi^{0} \nu \bar{\nu}$ is predicted in Table 2. As shown in fig. $1 \mathrm{c}, \sin ^{2} \beta$ is only weakly correlated with $\sin ^{2} \gamma$, measurable from the $\mathrm{CP}$ asymmetry in charged or neutral $B$-decays into a final state, containing a $D$-meson and a Kaon, which is not a CP-eigenstate [10].

We expect that none of the CP asymmetries that we have mentioned so far will be affected in any significant way by supersymmetric loop corrections in the decay amplitudes, with the possible exception of $a_{C P}\left(B_{d} \rightarrow \pi \pi\right)$. In this case, the dominant corrections should come from supersymmetric penguin diagrams and would be erased from the asymmetry by the isospin analysis invented to get rid of the (badly known) gluon-mediated penguin 
diagrams of the SM. On the other hand, asymmetries that could be significantly affected, in a controllable way, by supersymmetric loop corrections in the decay amplitudes, from penguin or box diagrams, are $a_{C P}\left(B_{d} \rightarrow \phi K_{S}\right)$ and $a_{C P}\left(B_{s} \rightarrow \phi \eta\right)$. A new phase $\chi$ would be introduced through the decay amplitude [11]:

$$
a_{C P}\left(B_{d} \rightarrow \phi K_{S}\right)=-\sin 2\left(\beta+\phi_{B_{d}}+\chi\right), \quad a_{C P}\left(B_{s} \rightarrow \phi \eta\right)=-\sin 2\left(\phi_{B_{s}}+\chi\right)
$$

that would make them deviate from $a_{C P}\left(B_{d} \rightarrow \psi K_{S}\right)$ and $a_{C P}\left(B_{s} \rightarrow \psi \phi\right)$ respectively, as given in (24).

\section{References}

[1] L.J. Hall and L. Randall, Phys. Rev. Lett. 652939 (1990);

M. Dine, R. Leigh and A. Kagan, Phys. Rev. D48 4269 (1993);

Y. Nir and N. Seiberg, Phys. Lett. B309 337 (1993);

P. Pouliot and N. Seiberg, Phys. Lett. B318 169 (1993);

M. Leurer, Y. Nir and N. Seiberg, Nucl. Phys. B398 319 (1993);

M. Leurer, Y. Nir and N. Seiberg, Nucl. Phys. B420 319468 (1994);

D. Kaplan and M. Schmaltz, Phys. Rev. D49 3741 (1994);

A. Pomarol and D. Tommasini, CERN-TH/95-207;

L.J. Hall and H. Murayama, Phys. Rev. Lett. 753985 (1995);

P. Frampton and O. Kong, IFP-725-UNC (1996);

E. Dudas, S. Pokorski and C.A. Savoy, Saclay T95/094, hep-ph/9509410;

C. Carone, L.J. Hall and H. Murayama, LBL-38047 (1995);

Y. Kawamura, H. Murayama and M. Yamaguchi, Phys. Rev. D51 1337 (1995);

N. Arkani-Hamed, H.-C. Cheng and L. J. Hall, LBL 37893 (1995); LBL 37894 (1996);

Z. Berezhiani, INFN FE 06-96, hep-ph/9609342.

[2] R. Barbieri, G. Dvali, and L. J. Hall, Phys. Lett. B377, 76 (1996);

R. Barbieri and L. J. Hall, LBL-38381, hep-ph/9605224, to appear in Nuovo Cimento $A$.

[3] R. Barbieri, L. J. Hall and A. Romanino, LBL-39488, hep-ph/9610449, to appear in Nucl. Phys. B..

[4] L. J. Hall and A. Rasin, Phys. Lett. B315 164 (1993).

[5] H. Leutwyler, CERN-TH/96-25, hep-ph/9602255 
[6] R. Barbieri and L. J. Hall, Phys. Lett. B338, 212 (1994).

[7] S. Bertolini, F. Borzumati, A. Masiero and G. Ridolfi, Nucl. Phys. B353 591 (1991);

A. Ali, G. Giudice and T. Mannel, Zeit. f. Phys. C67, 417 (1995);

T. Goto, Y. Okada, Y. Shimizu and M. Tanaka, KEK-TH-483, hep-ph/9609512;

T. Goto, T. Nihei and Y. Okada, Phys. Rev. D53, 5233 (1996);

P. Cho, M. Misiak and D. Wyler, Phys. Rev. D54, 3329 (1996);

J. Hewett and J. Wells, SLAC-PUB-7290, hep-ph/9610323.

[8] I. Bigi, in Results and Perspectives in Particle Physics, La Thuile, March 1994, p. 137

[9] G. Buchalla, A. Buras and M. Lautenbacher, Rev. Mod. Phys. 681125 (1996).

[10] I. Dunietz, Phys. Lett. B270, 75 (1991);

M. Gronau and D. Wyler, Phys. Lett. B265,172 (1991).

[11] N. Deshpande, B. Dutta and S. Oh, Phys. Rev. Lett. 774494 (1996)

Y. Grossman and M. Worah, SLAC-PUB-7351, hep-ph/9612269 